\title{
Endometrial Sampling Performed by Gynecological Residents in Training
}

\author{
Osama Sadkeak Bajouh1, Ohoud Al-Shamrany', Ahmad H. Abduljabbar², \\ Hassan S. O. Abduljabbar ${ }^{1}$ \\ ${ }^{1}$ Department of Obstetrics \& Gynecology, Medical College, King Abdulaziz University, Jeddah, KSA \\ ${ }^{2}$ Department of Obstetrics \& Gynecology, Baterji Medical College, Jeddah, KSA \\ Email: dr bajoouh@yahoo.com, dr.ohoud@hotmail.com, cenam5@hotmail.com, profaj17@yahoo.com
}

Received 16 September 2013; revised 18 October 2013; accepted 26 October 2013

Copyright (C) 2014 by authors and Scientific Research Publishing Inc.

This work is licensed under the Creative Commons Attribution International License (CC BY). http://creativecommons.org/licenses/by/4.0/

CC) (i) Open Access

\begin{abstract}
Objectives: Endometrial sampling became the first choice in screening endometrial pathology. The technique is accurate and simple. The aim is to evaluate the use of endometrial sample in outpatients' clinic as a routine by LEVEL of gynecological resident in training. Methods: A retrospective study of 463 patients seen by the residents at KAUH. A detailed history was obtained from medical records file. Reasons for endometrial sampling were divided into 6 categories, including screening, PMB, Menorrhagia or AUB, bulky uterus and cervical lesion. Endometrial sample was obtained using Pipelle. Results: Out of 463 patients, 128 had en-dometrial sampling as screening (27.6\%), 84 had PMB (18.1\%), 91 Menorrhagia (19.7\%), 108 AUB (23.3\%), 20 bulky uterus (4.3\%) and 32 cervical lesion (6.9\%). In $30 \%$ of cases the sample insufficient, diagnosis in $54.2 \%$ benign, endometrial hyperplasia (6.0\%) 19 diagnosed (endometrial cancer) $(4.1 \%)$ in $(5.6 \%)$ had endometritis. $70 \%$ of patients saved had a D\&C. Conclusion: Endometrial biopsy is found to be accurate, easy, and safe and can be done by LEVEL of gynecological residents, accuracy of $70 \%$ of cases, additional endometrial assessment undertaken if diagnosis is not made.
\end{abstract}

\section{Keywords}

Endometrial; Endometrial Biopsy; Outpatient

\section{Introduction}

Abnormal uterine bleeding accounts for $20 \%$ of visits to the gynecological clinic [1]. The most common pathological conditions cause abnormal vaginal bleeding, uterine leiomyoma's, endometrial polyps, and adenomyosis. Other less frequent conditions cause endometritis and serious but infrequent uterine cancers [2]. 
Exclusion of cancer or hyperplasia and making an accurate diagnosis is a challenge for the gynecologists. An endometrial biopsy (EMB) has been used extensively as an office procedure to obtain a sample of the endometrium. This sample is sent to pathology for analysis to help make the diagnosis [3].

Transvaginal ultrasound and sono-hysterography for selective patients has been used and it is a safe, non-invasive and, inexpensive method of triaging patients with abnormal uterine bleeding in order to determine which patients require no further evaluation, direct endometrial sampling using office hysteroscopy might be used in some cases when pathology is thought to be focal [4].

Endometrial pathology has been identified and diagnosed by using of several methods of biopsy such as Pipelle, Tao Brush, and Uterine Explora Curette. Endometrial sampling has gained popularity as an alternative to more invasive procedures such as fractional D\&C [5].

Endometrial sampling became the first choice in screening and evaluating women with bleeding or as screening methods for endometrial pathology. Endometrial sampling is an "office" procedure that used as method of endometrial assessment. It has gained widespread acceptance in gynecological practice. The technique is accurate and simple. The aim is to evaluate the use of endometrial sample in the outpatient clinic as a routine for endometrial assessment by LEVEL of gynecological resident in training.

\section{Subjects and Methods}

This a retrospective study of 463 patients presented to gynecological clinic seen by the residents at King Abdulaziz University Hospital from February 2010 till May 2011, all patients had an indication for endometrial sampling.

The inclusion criteria were age more than 40 years. Had one of the following indications for endometrial sampling, screening, PMB, menorrahgia, AUB, bulky uterus, and cervical lesion, Not diagnosed as endometrial cancer previously, and had endometrial sample using Pipelle endometrial sampler (Laboratories CCD, Paris, France) by LEVEL of gynecological residents.

The exclusion criteria age less than 40 or not from the 6 category of indication, diagnosed as endometrial cancer previously and had endometrial sampling using other than Pipelle endometrial sampler or by any health care provider other than a gynecological residents.

A detailed history of the patient was obtained from the medical records file. The reason for endometrial sampling was recorded and divided into 6 category, screening for patients asymptomatic but with high risk of endometrial cancer, patients with post menopausal bleeding, patients with Menorrhgia (heavy periods) or abnormal uterine bleeding, patients with bulky uterus and with cervical lesion.

Endometrial biopsy was done during the last week of a regular cycle. For irregular cycle it was done any time when the lady was not bleeding heavily. Endometrial sample was obtained using Pipelle sampler.

Pipelle sampler was used. Cusco's speculum was used to visualize the cervix. Pipelle was gently inserted into the cervical canal past the endocervix into the uterine cavity to the funds then sample obtained. If sample looked inadequate a second attempt was made after pushing the plunger and we used a double sampling technique with the same devise. In cases where insertion of pipelle is not possible, anterior lip of the cervix was steadied with volsellum forceps.

Endometrial sample sent to histopathology for assessment.

\section{Statistical Analysis}

The Statistical Package for the Social Sciences (SPSS) 15.0 software was used to analyze data.

\section{Results}

Out of total 463 patients, 128 patients had endometrial sampling as screening these patients were asymptomatic or patients with thick endometrium or patients going to be on hormonal replacement therapy or they already on it (27.6\%),

Eighty four patients had PMB (18.1\%), and 91 patients had heavy periods Menorrhgia (19.7\%).

In 108 patients, they had other type of bleeding abnormal uterine bleeding (23.3\%). Bulky uterus was the indication in 20 patients (4.3\%) and cervical lesion in 32 patients (6.9\%) (Table 1).

In $30 \%$ of cases the sample was reported to be insufficient for diagnosis. $28.9 \%, 19.9 \%, 5.4 \%$ were prolifera- 
tive, secretary, atrophic. Endometrial hyperplasia was diagnosed in 28 patients which account for (6.0\%), and endometrial cancer in 19 patients (4.1\%). and in 26 patients chronic endometritis was diagnosed (5.6\%) in Table 2.

\section{Discussion}

Dilatation and curettage is known standard method of endometrial assessment but the procedure have a limitation in form of it requires general anesthesia and has complications such as uterine perforation, hemorrhage and infection. It is also known that this method obtains tissue from less than $50 \%$ of the uterine cavity in $60 \%$ of procedures, and is not a form of treatment [6].

Endometrial sampling has a number of advantages compared with dilation and curettage it is an outpatientprocedure performed without anesthesia require no cervical dilation and no risk of uterine perforation, fast, simple and not expensive with adequate sample [7].

Numerous studies have shown that the endometrium is adequately evaluated with office sampling techniques. A sample can be obtained in 90 percent or more of patients [8]. It is important to remember, however, that all sampling devices do not provide visualization of the uterine cavity. Thus, they perform better when pathology is global rather than focal, e.g., a polyp.

Primary care physicians and gynecologists are aware that endometrial sampling is an important diagnostic tool in investigating and screening women above the 40 and, it has replaced D\&C as the first-line diagnostic test because it has similar accuracy, yet is safer, quicker, and more convenient [9].

In our study it was found that in screening of women above the age 40 years by gynecological residents in training trained to performed endometrial sample using Pipelle, $70 \%$ of patients had a definitive diagnosis

Table 1. Indication of endometrial sample.

\begin{tabular}{ccc}
\hline & Number of patients & Percentage \\
\hline Screening & 128 & 27.6 \\
PMB & 84 & 18.1 \\
Menorrhgia & 91 & 19.7 \\
AUB & 108 & 23.3 \\
Bulky Uterus & 20 & 4.3 \\
Cervical lesion & 32 & 6.9 \\
Total & 463 & 100 \\
\hline PMB post menapousal bleeding; AUB abnormal utrine bleeding.
\end{tabular}

Table 2. Histopathologiacl results of endometrial sample.

\begin{tabular}{ccc}
\hline & Number of patients & Percentage \\
\hline Insufficient & 139 & 30 \\
Proliferative & 134 & 28.9 \\
Secretary & 92 & 19.9 \\
Atrophic & 25 & 5.4 \\
Hyperplasia & 28 & 6.0 \\
Cancer & 19 & 4.1 \\
Chronic & 26 & 5.6 \\
Endometritis & 463 & 100 \\
Total & & \\
\hline
\end{tabular}


54.2\% were benign, endometrial hyperplasia (6.0\%) 19 patients diagnosed as endometrial cancer (4.1\%) and in (5.6\%) patients chronic endometritis was diagnosed.

This means that $70 \%$ of patients were saved to have a D\&C. In $30 \%$ of cases the sample was not adequate for diagnosis these women needed a proper assessment such as hysteroscopy and D\&C.

It has been reported that in general practice and in gynecological out patient's clinic that more than $70 \%$ of attempted samples of endometrium was successfully obtained [10].

In our study sampling was found to be easy to obtained require no anesthesia or analgesia, and no woman experienced more than slight discomfort during the procedure and there were no adverse effects except that very few cases have been reported to have an occasional vasovagal episodes. When sampling was not possible or the sample was Insufficient the patients would be referaed to have pelvic ultrasound and to be admitted for to have hysteroscopy and D\&C.

The Pipelle sampler of endometrium has been carefully evaluated since its development in 1984. It is generally said that Pipelle biopsies and D\&C have a nearly equal level of success in widespread endometrial lesions; Pipelle biopsies provide limited diagnostic accuracy in cases with focal pathologies [11].

In some countries including KSA there are many private clinic not run by level of consultant but by either specialist and residents and some of them might jump to to D\&C for assessment of endometrium.

If they have very simple training to do office endometrial sample that would save a lot patients unnesseary opration I mean D\&C.

\section{Conclusion}

Outpatient Pipelle endometrial biopsy is found to be accurate, easy and safe and can be done by LEVEL of gynecological residents in training with accuracy of $70 \%$ of cases; additional endometrial assessment should be undertaken if the diagosis is not made.

\section{References}

[1] Ashley Hill, D. (2009) Abnormal Uterine Bleeding: Avoid the Rush to Hysterectomy. Journal of Family Medicine, 58, 136-142.

[2] Pitkin, J. (2007) Dysfunctional Uterine Bleeding. BMJ, 334, 1110-1111. http://dx.doi.org/10.1136/bmj.39203.399502.BE

[3] Hernández, J.A., Franco, M.E., Mendizábal, D.P., Broca, Y.B. and Escoto, A.F. (2009) Evaluation of Postmenopausal Uterine Bleeding by Endometrial Biopsy In-Office Hysteroscopy vs Endometrial Biopsy with Manual Vacuum Aspiration in the Office. Preliminary report. Ginecología y Obstetricia de México, 77, 504-507.

[4] Steven, R.G. (2006) Abnormal Uterine Bleeding: The Role of Ultra Sound. Radiologic Clinics of North America, 44, 901-910.

[5] Shams, G. (2012) Comparison of Pipelle De Cornier with Conventional Dilatation and Curettage in Terms of Patients' Acceptability. Journal of Postgraduate Medical Institute, 26, 418-421.

[6] Fazio, S.B. and Ship, A.N. (2007) Abnormal Uterine Bleeding. Southern Medical Journal, 4, 376-382.

[7] Cooper, J.M. and Erickson, M.L. (2000) Endometrial Sampling Techniques in the Diagnosis of Abnormal Uterine Bleeding. Obstetrics and Gynecology Clinics of North America, 27, 235-244. http://dx.doi.org/10.1016/S0889-8545(00)80018-2

[8] Dijkhuizen, F.P., Mol, B.W., Brálmann, H.A. and Heintz, A.P. (2000) The Accuracy of Endometrial Sampling in the Diagnosis of Patients with Endometrial Carcinoma and Hyperplasia: A Meta-Analysis. Cancer, 89, 1765. http://dx.doi.org/10.1002/1097-0142(20001015)89:8<1765::AID-CNCR17>3.0.CO;2-F

[9] Samson, S. and Gilmour, D. (2002) Who Needs an Endometrial Biopsy? Canadian Family Physician, 48, 885-887.

[10] Clark, T.J., Mann, C.H., Shah, N., Khan, K.S., Song, F. and Gupta, J.K. (2001) Accuracy of Outpatient Endometrial Biopsy in the Diagnosis of Endometrial Hyperplasia. Acta Obstetricia et Gynecologica Scandinavica, 80, 784-793. http://dx.doi.org/10.1034/j.1600-0412.2001.080009784.X

[11] Kazandi, M., Okmen, F., Ergenoglu, A.M., Yeniel, A.O., Zeybek, B., Zekioglu, O. and Ozdemir, N. (2012) Comparison of the Success of Histopathological Diagnosis with Dilatation-Curettage and Pipelle Endometrial Sampling. Journal of Obstetrics \& Gynaecology, 32, 790-794. http://dx.doi.org/10.3109/01443615.2012.719944 